\title{
PECVD grown SiN photonic crystal micro-domes for the light extraction enhancement of GaN LEDs
}

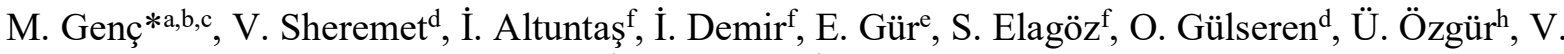 \\ Avrutin $^{\mathrm{h}}, \mathrm{H}$. Morkoç ${ }^{\mathrm{h}}$ and A. Aydınlı ${ }^{\mathrm{g}}$ \\ aTyndall National Institute, Cork, Ireland; ' ${ }^{\mathrm{a}}$ Sabanc1 University, Department of Electronics \\ Engineering, İstanbul, Turkey; ${ }^{\circ}$ Ermaksan Optoelectronic R\&D Center, Bursa, Turkey; ${ }^{\text {B Bilkent }}$ \\ University, National Nanotechnology Research Center (UNAM), Ankara, Turkey; ${ }^{\mathrm{e} A t a t u ̈ r k}$

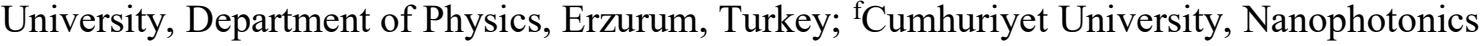 \\ Research and Application Center, Sivas, Turkey; gUludağ University, Department of Electrical and

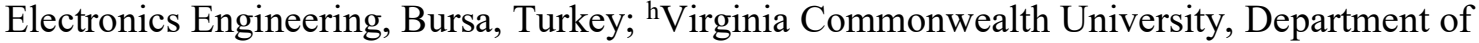 \\ Electrical and Computer Engineering, Richmond, VA, USA \\ muhammet.genc@tyndall.ie \\ atillaaydinli@uludag.edu.tr
}

\begin{abstract}
In this work, the effect of introducing a photonic crystal network of silicon nitride (SiN) micro-domes on the backside of silver coated gallium nitride (GaN) based light emitting diodes (LEDs) was studied. First, sapphire side of the top emitting LEDs, which is the bottom surface of the LEDs, is coated with silver (Ag). Light emitted towards the sapphire substrate is reflected upwards to the top surface and the amount of light extracted from the LED is expected to increase. In an alternative approach, SiN micro-domes forming a two dimensional photonic crystal, $2 \mu \mathrm{m}$ in diameter and $80 \mathrm{~nm}$ in height in average, are deposited on the light emitting surface of the device with a period of $2 \mu \mathrm{m}$. Coating the backside with $\mathrm{Ag}$ has increased the efficiency of a top emitting LED by $11 \%$. By introducing the SiN photonic crystal onto the Ag backside coated sample, total internal reflection is reduced via scattering and the amount of light emitted has been increased by $30 \%$ at $5 \cdot 10^{4} \mathrm{~mA} / \mathrm{cm}^{2}$. Integration of SiN micro-domes with Ag coating has significantly impacted light extraction which has been shown to increase the efficiency of GaN based LEDs. Fabrication process and the results are discussed in detail.
\end{abstract}

Keywords: Light extraction, $\mathrm{Si}_{3} \mathrm{~N}_{4}$ micro-domes, LED performance, light emitting diodes

\section{INTRODUCTION}

High brightness LEDs have been used in various applications including next generation displays such as augmented and virtual reality (AR $\backslash \mathrm{VR})$ headsets, micro-LED displays, communication, automotive lighting and general lighting for optoelectronics. Many research activities have been focused to achieve higher brightness LEDs with larger quantum efficiencies. One of the biggest problems limiting the brightness of LEDs is the total internal reflection, which reduces the photon removal efficiency of the LEDs [1]. Variety of design techniques have been studied to enhance light extraction efficiency, such as surface roughening of sapphire or ITO, chip forming (shaping), and the use of photonic crystals. In this context, the refractive indices of the sapphire substrate and the epitaxial layers grown on it, are very large relative to air. Refractive index of sapphire is $n=1.7$ at $450 \mathrm{~nm}$ wavelength and the refractive index of GaN layers is 2.25 . If we neglect the refractive indices of the possible thin InGaN and $\mathrm{AlGaN}$ layers in between, the Fresnel equations show that the exact total internal reflection angle is quite small, such that the light produced in the InGaN quantum wells can hardly escape the LED. Interfaces of GaN with air, sapphire with air, sapphire with GaN, emitting light from Fresnel cones is limited by total internal reflection. The light cone between $\mathrm{GaN}$ and air is shown schematically in figure 1. Various strategies have been developed to overcome the limitations of the light cone. One of the most commonly used methods is to overcome the cone limitation of light is by increasing the scattering between air and sapphire or air and GaN [2]. In this work, scattering was used by coating a thin film by adjusting of refractive index on $\mathrm{GaN}$ or sapphire and patterning the corresponding film. 


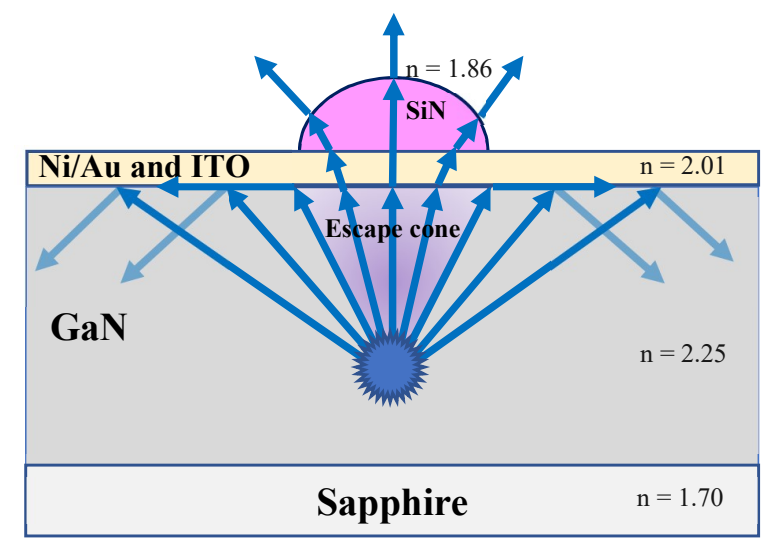

Figure 1. Escape cone and total internal reflection of light.

The refractive index of $\mathrm{SiN}$ thin films can be adjusted by changing the PECVD growth parameters such as gas flow rates and growth temperatures. The SiN thin film coated at the interface where scattering is achieved, is then patterned by photolithography to increase the light extraction efficiency [3].

\section{EXPERIMENTAL}

InGaN MQW based LED structures were grown by metal organic chemical vapor deposition (MOCVD) system on sapphire substrate. Epitaxial layers consist of $10 \mu \mathrm{m}$ undoped GaN grown by in situ nano-ELO technique, followed by a $4 \mu \mathrm{m}$ thick n-GaN $\left(3 \times 10^{18} \mathrm{~cm}^{-3}\right)$, a $60 \mathrm{~nm} \operatorname{In}_{0.01} \mathrm{GaN}_{0.99}$ stress compensation layers, a 7 step graded $\operatorname{In}_{\mathrm{x}} \mathrm{Ga}_{1-\mathrm{x}} \mathrm{N}(\mathrm{x}=0.04$ to 0.1 with $3 \mathrm{~nm}$ thickness for each step) electron injector layer, $6 \times 2 \mathrm{~nm} \operatorname{In}_{0.15} \mathrm{Ga}_{0.85} \mathrm{~N}$ quantum wells separated by $3 \mathrm{~nm}-$ thick $\mathrm{In}_{0.06} \mathrm{Ga}_{0.94} \mathrm{~N}$ barriers, and finally a $100 \mathrm{~nm}$ p-GaN $\left(4 \times 10^{17} \mathrm{~cm}^{-3}\right)$. Figure 2 shows the grown epitaxial structure. The step graded electron injector (SEI) is included in the structure to help to cool down the electrons prior to injection into the MQW region and thus mitigate the electron overflow [4].

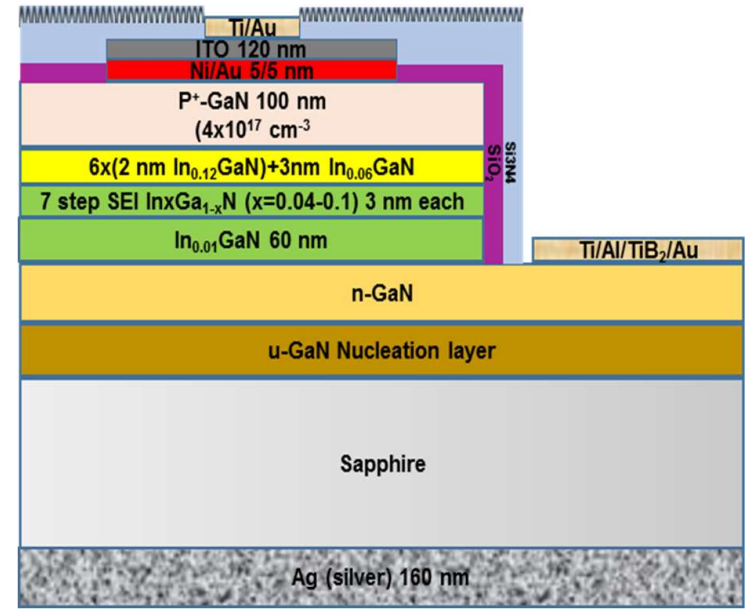

Figure 2. Cross-sectional view of LED structure

Fabrication of the LED device is as follows; square mesas of $1 \mathrm{~mm}^{2}$ were formed by ICP etching with $\mathrm{BCl}_{3}: \mathrm{Cl}_{2}$ gases. LEDs had interdigitated electrode patterns with distance from contact pads to the edge of etched mesa equals to $150 \mu \mathrm{m}$. Ohmic contacts to $\mathrm{n}-\mathrm{GaN}$ were formed using a $\mathrm{Ti}(30 \mathrm{~nm}) / \mathrm{Al}(50 \mathrm{~nm}) / \mathrm{TiB}_{2}(50 \mathrm{~nm}) / \mathrm{Au}(70 \mathrm{~nm})$ metal stack which was subjected to rapid thermal annealing (RTA) in $\mathrm{N}_{2}$ ambient at $\mathrm{T}=800^{\circ} \mathrm{C}$ for 30 second after electron beam evaporation. For current spreading layer and ohmic contact to p-GaN, a Ni(5 nm)/Au(5 nm)/ITO(120 nm) stack was used with Ti(30 $\mathrm{nm}) / \mathrm{Au}(50 \mathrm{~nm})$ contact pads on the ITO. ITO thin film was grown $120 \mathrm{~nm}$ on top of the p-GaN layer by RF sputtering. 
a)

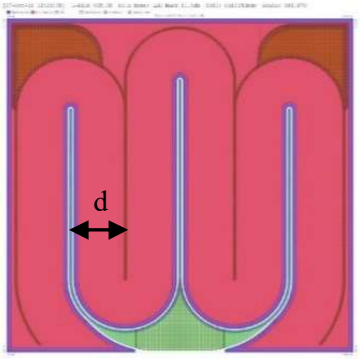

b)

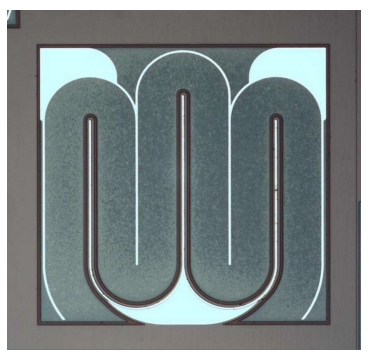

c)

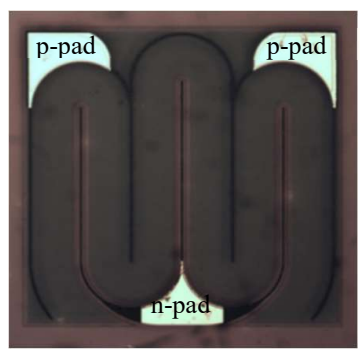

Figure 3. a) $1 \mathrm{~mm}^{2}$ single chip design (photomask) with $\mathrm{d}=150 \mu \mathrm{m}$, b) fabricated LED chip with pre-pass and silver, c) additional SiN micro-domes located everywhere except at the $\mathrm{p}$ - and $\mathrm{n}$ - contact pads.

In this study, the sample was fabricated and denoted as pre-passivated, pre-passivated and silver and lastly pre-passivated, silver and $\mathrm{SiN}$ micro-domes. The pre-passivation was employed with PECVD-deposited $\mathrm{SiO}_{2}$ of $250 \mathrm{~nm}$ thickness immediately after ICP mesa etching. After the metal deposition on the p-GaN side, Finally, $230 \mathrm{~nm} \mathrm{SiN}$ was grown by the PECVD technique on top of the $\mathrm{SiO}_{2}$ passivation layer. For pre-passivation and silver sample, then, $160 \mathrm{~nm}$ silver was deposited to collect escaping light from sapphire side. For light extraction improvement, micro-domes were formed with the assist of UV lithography followed by etching in buffered oxide etchant (BOE). Windows in SiN passivation layer were gained to access just the contact pads on the $\mathrm{n}-\mathrm{GaN}$ and contact pads on the current spreading ITO layers. Photomask design and optical microscope images of fabricated LED chips were shown in figure 3. Shematic of the fabricated photonic crystals and the optical microscope images taken after fabrication were given in figure 4. Electrical properties of fabricated LED dies were measured using a Keithley 2430 source and an HP 4142B monitor unit. Optical properties were measured by Instrument Systems spectrometer CAS 140CT. Ohmic contact resistivities were measured by using transmission line method (TLM) with linear and circular contact pad geometries. For linear TLM measurements, $75 \times 450 \mu \mathrm{m}^{2}$ contact pads were used with separations of 20,40,60,80, 100 and $150 \mu \mathrm{m}$. For circular TLM measurements inner contact pads had diameters of 50,80, 100 and $150 \mu \mathrm{m}$ with a constant ratio between outer and inner contact diameters of $2[5,6]$.

a)

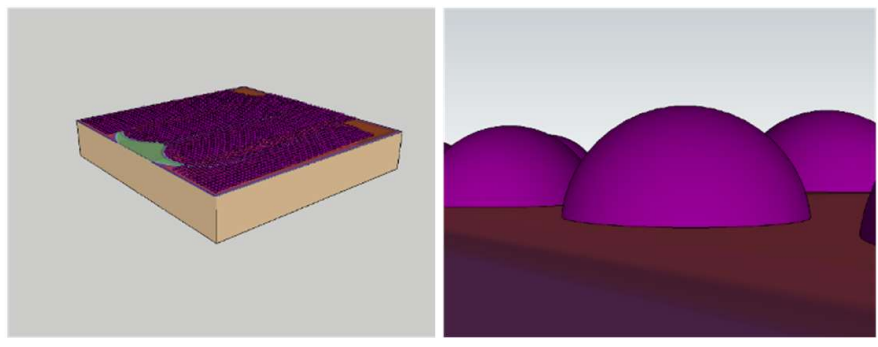

b)
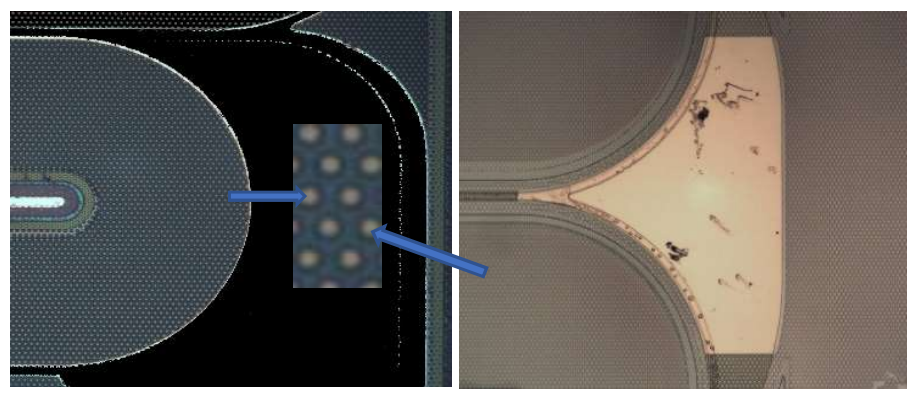

Figure 4. a) 3D drawings of SiN photonic crystals b) optical microscope pictures of 2D photonic crystals that was fabricated by using reflow method. Blue arrows point to $\mathrm{SiN}$ micro-domes. 


\section{RESULTS}

The shape and the heights of the micro domes of the photonic crystals were analyzed using a dektak surface profilometer. $2 \mu \mathrm{m}$ scanning stylus was chosen to measure hills and valleys.
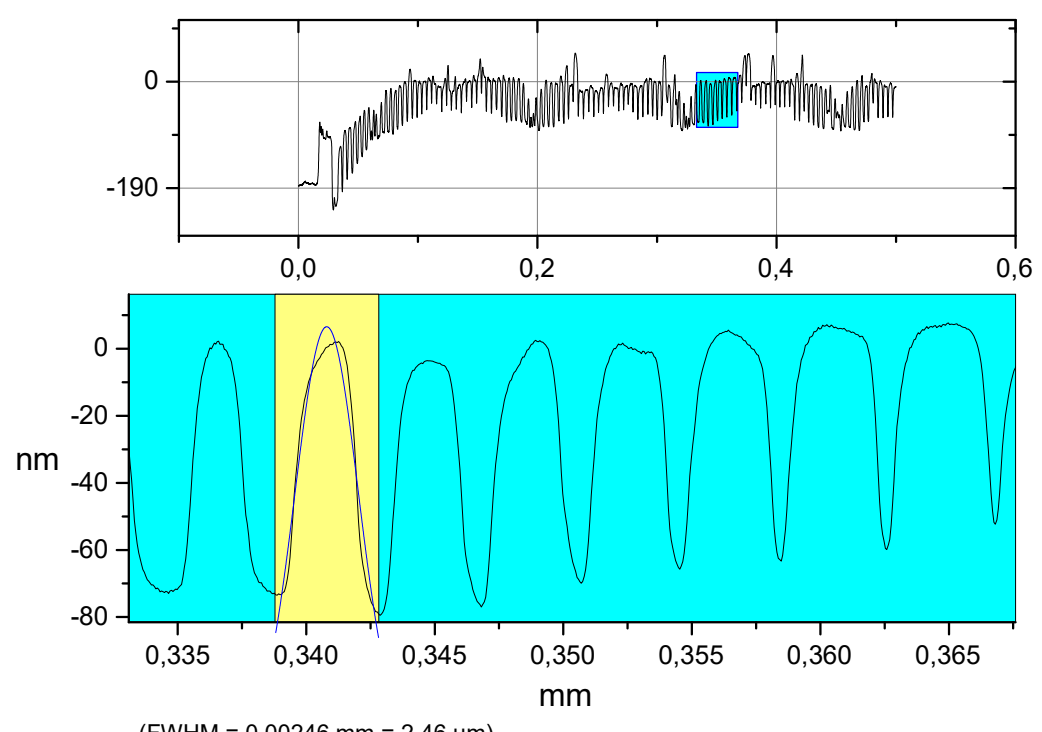

Figure 5. Surface profiles of photonic crystal micro-domes.

The heights of micro-domes were measured as $80 \mathrm{~nm}$ (average), while the width of micro-domes are $2.46 \mu \mathrm{m}$ with $2 \mu \mathrm{m}$ spacing between them. We note that micro-domes are uniform in size and uniformly distributed over the chip. As shown in figure 5. 
a)

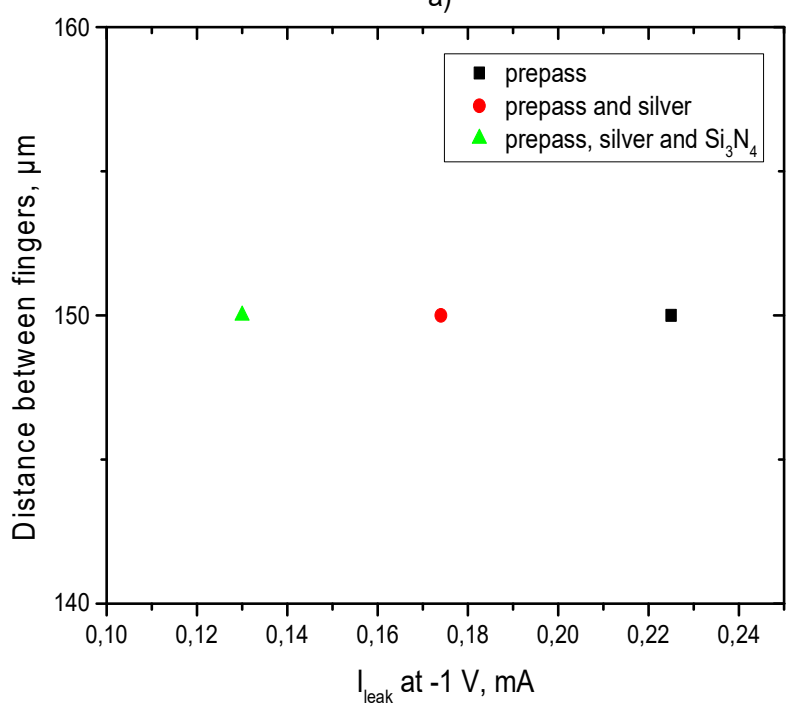

b)

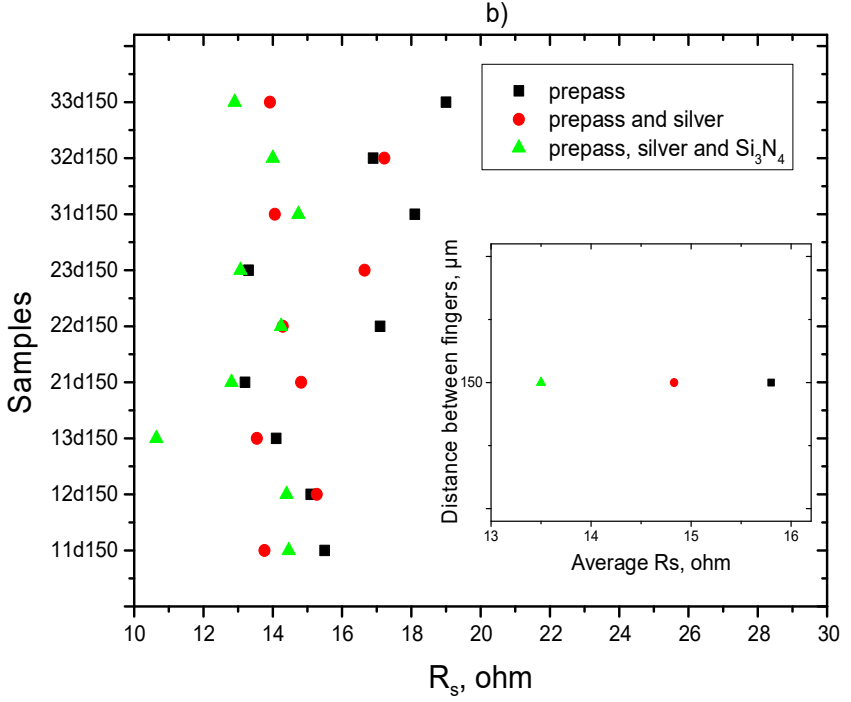

Figure 6. a) Leakage current values at -1 Volt and b) average series resistance values of different LED dies

Series resistance was obtained for the LED with pre-passivation, silver and SiN has been lowered by $14.6 \%$ compared to that of the pre-passivated sample. On the other hand, the leakage current for the same sample has decreased to $25.3 \%$ compared to that of the pre-passivation and silver sample (figure 6). It seems that the SiN passivation has improved the device performances. 


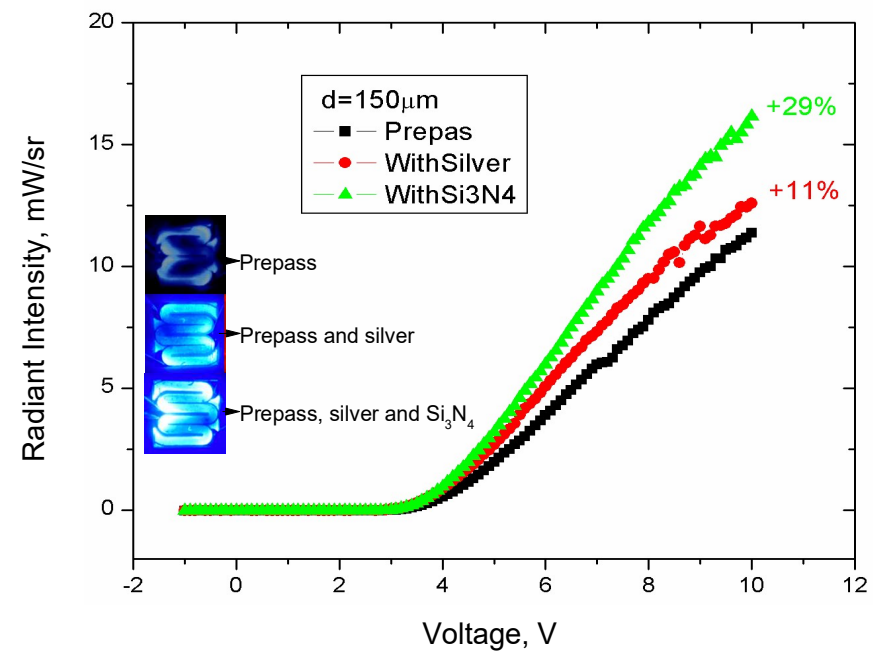

Figure 7. Radiant intensity values of device with pre-passivation, with silver and with $\mathrm{Si}_{3} \mathrm{~N}_{4}$ in terms of voltage

The total power values of the LED sample were increased by $29 \%$ for samples with $150 \mu \mathrm{m}$ finger spacing due to SiN micro-dome photonic crystals inclusion as shown in figure 7. Inset shows the microscope images of the LEDs when they are emitting. The brightness of the light increases gradually by using additional $\mathrm{Ag}$ back contact and $\mathrm{Si}_{3} \mathrm{~N}_{4}$ micro domes. It is clear from the figures that $\mathrm{SiN}$ micro-domes significantly improves the light extraction.

As a summary, photonic crystal design was used for situations where light emerges from the top [2]. In order to increase the emission of light, SiN photonic crystals were grown in PECVD system in $2.46 \mu \mathrm{m}$ diameter, $80 \mathrm{~nm}$ height and $2 \mu \mathrm{m}$ periods, and eventually pattern structures were formed by reflow method. It is clearly shows that additional silver and SiN, has significantly impacted of light extraction enhancement.

\section{ACKNOWLEDGEMENT}

This work is supported by Scientific and Technical Research Council of Turkey, (TUBITAK Grants no: 113 G042 and 115E109). We thank to Advanced Research Laboratory of Bilkent University and Ermaksan Optoelectronic R\&D Center for the use of fabrication and characterization equipments.

\section{REFERENCES}

[1] Kyeong-Jae Byeon et al., "Fabrication of two-dimensional photonic crystal patterns on GaN-based light-emitting diodes using thermally curable monomer-based nanoimprint lithography", Appl. Phys. Lett. 91, 091106 (2007).

[2] J. J. Wierer et al., (2005), III-Nitride LEDs with photonic crystal structures, Proc. of SPIE Vol. 5739.

[3] Kim et al., "Elimination of total internal reflection in GaInN light-emitting diodes by graded-refractive-index micropillars", Appl. Phys. Lett. 93, 2211112008.

[4] H. Morkoç, Handbook of Nitride Semiconductors and Devices, Vol. 1: Materials Properties, Physics and Growth, WILEY-VCH Verlag GmbH \&Co. KGaA, Weinheim, 2008.

[5] V. Sheremet et al. / "Two-step passivation for enhanced InGaN/GaN light emitting diodes with step graded electron injectors, Superlattices and Microstructures”, 113 (2018) 623-634.

[6] M. Genç et al., "Distributed contact flip chip InGaN/GaN blue LED; comparison with conventional LEDs", Superlattices and Microstructures 128 (2019) 9-13. 